\title{
1 ASER: Animal Sex Reversal database
}

2 Yangyang $\mathrm{Li}^{1, \#}$, Zonggui Chen ${ }^{2, \#}$, Hairong Liu ${ }^{3, \#}$, Qiming $\mathrm{Li}^{1}$, Xing $\mathrm{Lin}^{2}$, , Shuhui Ji

$3{ }^{3}$, Rui $\mathrm{Li}^{3}$, Shaopeng $\mathrm{Li}^{1}$, Weiliang $\mathrm{Fan}^{1}$, Haiping Zhao ${ }^{3}$, Zuoyan $\mathrm{Zhu}^{2}$, Wei $\mathrm{Hu}^{2,4}$, $4 \mathrm{Yu}$ Zhou ${ }^{1,5, *}$, Daji Luo Lu, $^{2,4, *}$

5

$6{ }^{1}$ State Key Laboratory of Virology, College of Life Sciences, Wuhan University,

$7 \quad$ Wuhan 430072, China

$8{ }^{2}$ State Key Laboratory of Freshwater Ecology and Biotechnology, Institute of 9 Hydrobiology, Innovation Academy for Seed Design, Chinese Academy of Science, 10 Wuhan 430072, China

$11{ }^{3}$ School of Basic Medical Science, Wuhan University, Wuhan 430071, China

$12{ }^{4}$ University of Chinese Academy of Sciences, Beijing 100049, China.

$13{ }^{5}$ Frontier Science Center for Immunology and Metabolism, Wuhan University, Wuhan 14 430072, China

$16 \quad$ Equal contribution.

$17 *$ Corresponding authors.

18 Email: huwei@ihb.ac.cn (Hu W), yu.zhou@whu.edu.cn (Zhou Y), luodaji@ihb.ac.cn 19 (Luo DJ)

Running title: ASER: Animal Sex Reversal Database

23 The numbers of words: 4321.

24 The numbers of figures: 4.

25 The numbers of tables: 1.

26 The numbers of supplementary figures: 1.

27 The numbers of supplementary tables: 3 .

28 The numbers of all the References: 40.

29 The numbers of References from 2014: 23. 
30 The counts of letters in the article title: 34.

31 The counts of letters in the running title: 34 .

32 The count of keywords: 5.

33 The counts of words in Abstract: 183. 


\section{Abstract}

35 Sex reversal, representing extraordinary sexual plasticity during the life cycle, not 36 only triggers reproduction in animals but also affects reproductive and endocrine 37 system-related diseases and cancers in humans. Sex reversal has been broadly 38 reported in animals, however, an integrated resource hub of sex reversal information is still lacking. Here, we constructed a comprehensive database named ASER by 40 integrating sex reversal-related data of 18 species from Teleostei to Mammals. We systematically collected 40,018 published papers and mined the Sex

42 Reversal-associated Genes (SRGs), including their regulatory networks, from 1,611 core papers. We annotated homologous genes and computed conservation scores for whole genomes across the 18 species. Furthermore, we collected 206 available RNA-seq data and investigated the expression dynamics of SRGs during sex reversal or sex determination processes. In addition, we manually annotated 551 ISH images of SRGs from the literature and described their spatial expression in the gonads. Collectively, ASER provides a unique and integrated resource for researchers to query and reuse organized data to explore the mechanisms and applications of SRGs in animal breeding and human health. The ASER database is publicly available at http://aser.ihb.ac.cn/.

52

KEYWORDS: Sex reversal; SRGs; Database; Omics; Conservation

54 
62

63

64

65

66

67

68

69

\section{Introduction}

Sex determination mechanisms in animals mainly include genetic sex determination (GSD) and environmental sex determination (ESD) [1]. In GSD, the primary sex of organisms is determined by genetics during fertilization, while organisms with ESD remain bipotential gonads until they perceive environmental stress to promote sex differentiation during ontogeny [2]. For many years, it was dogma in vertebrates in the field of sex determination that sex would be fixed for life after primary sex determination. After sex reversal was first reported in Aplocheilus latipes and natural sex reversal was found in Monopterus javanensis [3], it has been widely accepted that sex determination is amazingly plastic in vertebrates, especially in fish. This plasticity shows that sexual fate is not an irreversible process. Indeed, this reversible process leads to sex reversal, a redirection of the sexual phenotype during development [4]. Environmental factors can override genetic factors to redirect sexual fate in fish [5] and reptiles [6]. Sex reversal was found to be driven by diverse factors, such as genes, hormones, temperature, and social changes [7]. Unlike sex change, which implies a transition from the stabilized sex to the opposite sex, sex reversal occurs during gonadal development, including the initiation phase and maintenance phase of sex determination [4].

Specifically, sex reversal has been studied in fish, reptiles, birds, amphibians and even in mammals. In fish, gonadal differentiation is roughly divided into two groups: hermaphroditic and gonochoristic [5]. Hermaphroditic species undergo sex reversal during their lifetime and include 3 strategies: female-to-male (protogynous), male-to-female (protandrous), or bidirectional (serial) sex change [8]. Taking Monopterus albus as an example, an individual is female from the embryonic stage to first sexual maturity, then enters an intersex state, and later develops into a male [9]. Additionally, some hermaphroditic species undergo socially cued female-to-male sex reversal, whereby the removal of the dominant male induces sex reversal in a resident female, such as Thalassoma bifasciatum [10]. Among gonochoristic fish, sex reversal 
90 is a synergistic result of both GSD and ESD [11]. For example, Cynoglossus 91 semilaevis is a gonochoristic fish with a female heterogametic sex determination

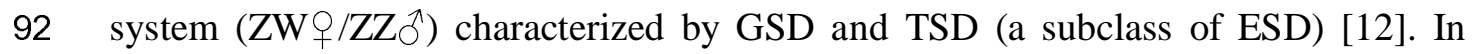
93 many reptiles, including Trachemys scripta, gonadal sex is determined by the 94 environmental temperature during egg incubation [13]. However, estrogens, including 95 estradiol-17 $\beta$, have also been proven to participate in the sex determination of $T$. 96 scripta [14]. Sex reversal in birds such as Gallus gallus, is mainly related to 97 alterations in sex steroid hormone action, especially estrogens [15]. Amphibians also 98 show plasticity in sex determination, influenced by estrogens, androgens [16], and sometimes by temperature [17]. Sex determination in mammals has been reported to 100 depend on three processes: chromosome determination (XX or XY), appropriate 101 pathway of gonadal differentiation, and accurate development of secondary sexual characteristics [18]. Disrupting any of these three steps of gonadal differentiation can lead to aberrant sex determination. In Homo sapiens, the frequencies of $\mathrm{XX}$ and $\mathrm{XY}$ sex reversal are 1/20,000 and 1/3,000, respectively, and most of these cases are caused by translocations of the sex-determining $S R Y$ gene [19]. Although sex reversal has been broadly reported among vertebrates, the molecular events underlying sex reversal remain poorly understood, limited by the lack of integrated omics data across species.

Although there are several reproduction-related resources, such as GUDMAP [20], GonadSAGE [21] and ReproGenomics Viewer [22], an integrated and dedicated database for the community studying sex determination and differentiation is missing. The GUDMAP database is a comprehensive gene expression dataset of the 113 developing genitourinary system in mouse with both in situ and microarray data. 114 GonadSAGE is a serial analysis of gene expression database for male embryonic 115 gonad development in mouse. The ReproGenomics Viewer is a cross-species database 116 of omics data such as RNA-seq and ChIP-seq for tissues related to reproduction, such 117 as gametogenesis, in 9 model organisms. Here, we developed the Animal Sex 118 Reversal database (ASER), the first functional genomics hub for sex reversal to our 119 best knowledge. The main works of ASER can be roughly divided as follows: (1) We 
screened 18 important and typical species with sex reversal phenomena from Teleostei to Mammalia, including Betta splendens, Cyprinus carpio, Cynoglossus semilaevis, Danio rerio, Equus caballus, Epinephelus coioides, Lates calcarifer, Monopterus albus, Oryzias latipes, Oreochromis niloticus, Paralichthys olivaceus, Thalassoma bifasciatum, Xenopus laevis, Gallus gallus, Bos taurus, Mus musculus, and Homo sapiens, and summarized the major inducements of sex reversal or common approaches used to manipulate sex in these species (Table 1). (2) We compiled a list of the most common genes or drugs related to sex reversal. Then, we collected and analyzed PubMed literature to mine the Sex Reversal-associated Genes (SRGs) and obtained their regulatory networks. Meanwhile, we gathered protein-protein interaction networks related to SRGs from the STRING database. (3) To facilitate users comparing the homology of SRGs in different species, we collected or assembled the gene annotations for the 18 species, identified homologous genes, computed the basewise conservation scores across these species, and identified conserved motifs for orthologous gene groups. (4) We systematically processed available RNA sequencing (RNA-seq) data and provided gene expression dynamics during sex reversal between females and males or different developmental stages. A user-friendly genome browser was customized to visualize these genome-wide data. (5) We collected and annotated available in situ hybridization and immunocytochemistry (ISH, FISH, and ICH) data to display the spatial expression of SRGS in the gonads. In conclusion, our ASER database provides comprehensive and systemic integration of sex reversal related data, and we believe that this open resource will greatly promote research on the mechanisms of sex reversal.

\section{Data collection and database content}

\section{Framework of ASER}

An overview of the ASER database and web server is shown in Figure 1. The ASER database contains information for 18 sex reversal species, SRG regulatory networks, 
149 homology alignment, and (fluorescence) in situ hybridization and 150 immunocytochemistry (ISH, FISH, and ICH) images of SRGs. The preprocessed data 151 was managed with the MySQL database. Django-based applications were developed 152 to provide a user-friendly interface including an embedded genome browser for 153 visualizing genome-wide data. The key workflows, tools, and processed data are 154 summarized in Figure S1A-B and described in detail below.

\section{Data sources}

157 SRG information and their regulatory networks were curated from PubMed literature. 158 All genome sequences and species information used in this database were 159 downloaded from NCBI public databases. All raw sequencing data were downloaded 160 from the Sequence Read Archive (SRA) of NCBI. The sets of RNA-seq data were organized by species, gonad developmental stages, and temperature (Table S1). In addition, we retrieved images related to sex reversal from the OPENi (https://openi.nlm.nih.gov/) and ZFIN databases [23].

\section{SRG mining}

We retrieved thousands of articles from PubMed by querying species and functional keywords (e.g., sex reversal). First, the abstracts and full texts of these articles were collected by text crawler technology. The full texts of non-open access papers were obtained through the library portal of Wuhan University. Next, we separated a chunk of continuous text into separate words, and carried out word stemming to remove plural and different tenses. Then, we removed stop words such as such as "the", "is", and "however". Finally, we counted the frequency of the words from the literature and manually filtered out some high-frequency but irrelevant words such as 174 "masculinizing", “ovotestes", "pseudomale", "hermaphrodite", and "gynogenesis" 175 into a blacklist until most of the high-frequency words were gene symbols and drug 176 names. The remaining words related to genes and drugs were manually added to the 177 wordlist (Table S2).

178 We retained 1,611 papers that contained the words in the wordlist and manually 
179 read them with notations about SRG regulation (Table S3). Finally, we found 258

180 SRGs, 6 drugs and 11 hormones, which were validated to be functional in sex reversal

181 in different species, and constructed the regulatory networks of SRGs. We next

182 predicted another 498 genes that were homologous with those SRGs in the 18 species

183 (Figure S1C). Furthermore, protein-protein interaction (PPI) networks of SRGs were

184 extracted from the STRING database [24].

\section{RNA-seq data processing}

187 The data quality of the collected RNA-seq data was assessed using FastQC 188 (http://www.bioinformatics.babraham.ac.uk/projects/fastqc/), and the adapters and 189 low-quality bases in raw reads were removed using Trim Galore 190 (http://www.bioinformatics.babraham.ac.uk/projects/trim_galore/). Filtered reads 191 were aligned to the genome using STAR [25] in end-to-end mode. The primary 192 alignments were retained through SAMtools [26]. Gene expression quantification in 193 FPKM (Fragments Per Kb of exon per Million mapped fragments) was computed 194 using StringTie [27]. Differential expression analysis was performed using DESeq2 195 [28].

\section{Transcriptome assembly}

High-quality reads were de novo assembled using StringTie [27] with default parameter settings. The longest ORFs were predicted in assembled transcripts using TransDecoder.LongOrfs (https://help.rc.ufl.edu/doc/TransDecoder). DIAMOND [29] was used to collect homologous evidence of identified ORFs from the UniProt database (https://www.uniprot.org/). The potential coding regions were further refined by TransDecoder.Predict. Finally, a GFF3 file based on the coding regions of the reference genome was generated through the cdna_alignment_orf_to_genome_orf.pl function in TransDecoder.

\section{Homology alignment}

208 Orthologous groups of SRGs were identified among all sex reversal species using the 
BLAST [30] all-v-all algorithm in OrthoFinder [31]. Conserved motifs of orthogroups

210 were predicted using MEME [32]. Comparisons between conserved motifs and

211 known motifs were performed using Tomtom [33]. Species tree was constructed

212 according to the species taxonomy on NCBI. Meanwhile, the evolutionary

213 relationship was verified by OrthoFinder using the STAG [34] algorithm and rooted

214 using the STRIDE [35] algorithm.

215 For the orthologue tracks in a reference species, the homologous genes in other

216 species were mapped to the reference genome using Blat [36]. Alignments with

217 sequence identity larger than or equal to $60 \%$ were retained, and the maximum intron

218 size was set to $450,000 \mathrm{bp}$.

219 For the conservation track, pairwise alignments between genome sequences were 220 built using LASTZ [37], and MULTIZ [38] was then used to construct multiple 221 alignments, based on which the conservation scores were calculated using phyloP 222 from the PHAST package [39].

223

\section{Image collection and annotation}

225

226

227

228

229

230

231

232

233

234

235

236

237

We collected available (fluorescence) in situ hybridization and immunocytochemistry (ISH, FISH and ICH) data related to SRGs from the OPENi and ZFIN databases. The images were classified by gene, differentiation status, developmental period, and gender. We manually added descriptions for those images based on the original figure legends and articles.

\section{Web interface and usage}

ASER is a user-friendly database, and all the contents are interactive and dynamic. There are five main functional modules, including SPECIES, IMAGE, SRG, GENE and BROWSER. In addition, the SEARCH module was developed to display and interconnect different kinds of data in other modules.

For the "SPECIES" module, the evolutionary tree constructed for the 18 sex reversal species is displayed on the main page (Figure 2A). The reported 

inducements of sex reversal or common approaches used to manipulate sex in each species are displayed on this page, including natural processes, genetic abnormality (e.g., amh overexpression), administration of exogenous hormones or drugs (e.g.,

241 17 $\alpha$-methyltestosterone), temperature changes during gonadal differentiation, and manipulation of social factors. The literature supporting this information is also provided. Users can click on any species to obtain detailed descriptions and genome information for this species (Figure 2B).

For the "IMAGE" module, we summarized the morphological characteristics of zebrafish and mouse ovary at different developmental stages to help users better understand the content of this module (Figure 2C). The (fluorescence) in situ hybridization and immunocytochemistry (ISH, FISH and ICH) data related to specific SRGs can be queried in different ways by species, gene, differentiative stage, and gonad. Detailed descriptions of images are shown to help users understand the spatial distribution of SRGs in the gonads (Figure 2D).

The "SRG" module includes word cloud, regulatory and PPI networks, and search pages. The word cloud figure is dynamically presented by species with hyperlinks on the nodes (Figure 3A). When the user clicks one node, the original references and additional actions for more information will be shown under the figure. For any validated SRG, ASER allows users to obtain its regulators (including genes, hormones, drugs), targets, and the associated modes of regulation (Figure 3B). At the same time, PPI networks of these SRGs in different species are also displayed (Figure 3C), in which the colors of the edges are used to distinguish known interactions (experimentally_determined_interaction, database_annotated), predicted interactions (neighborhood_on_chromosome, gene_fusion, phylogenetic_cooccurrence), and other types (homology, coexpression, automated_textmining). In addition, the search page provides an interface for a specific SRG to show its regulatory network and more detailed information, such as tissue, developmental stage, and literature evidence (Figure 3D-E).

The "GENE" module provides different kinds of data for any annotated gene, 
268

269

270

271

272

273

274

275

276

277

278

279

280

281

282

283

284

285

286

287

288

289

290

291

292

293

294

295

296

corresponding to the query gene are shown in the search page by species and gene symbol (Figure 4A). Detailed information for a specific query gene includes its orthogroup in all species, predicted motifs, and similar known motifs (Figure 4B). The orthologous genes and 18-way conservation scores for the query gene can be inspected in BROWSER tracks (Figure 4C). Detailed alignment information can be obtained and downloaded by clicking on the track. In addition, the gene expression quantifications in FPKM across different stages, tissues, and conditions are shown as bar plots and in detail as tables (Figure 4D). The RNA-seq signal profiles are displayed in BROWSER, and the tracks can be customized easily, including color, scale, height, and etc. For any species, the available tracks can be dynamically selected or unselected. For example, in Danio rerio, a subset of RNA-seq tracks are shown for the sox9 gene during sex reversal (Figure 4E).

\section{Discussion}

Studies on sex reversal have been especially useful in helping redefine the concept of sex determination. There are diverse master sex-determining genes reported in different species. In addition, genes previously known to be involved in sex determination or differentiation are emerging as potential key components of sex reversal in other vertebrates [40]. Therefore, studies in different species continue to reveal genes with unexpected roles in sex reversal, and their homologs in other vertebrates also deserve investigation. ASER fills the gap of the sex reversal database by integrating diverse information at different levels for the 18 species with sex reversal, including curated SRGs, RNA-seq data, image data, and conservation data.

For any collected sex reversal species, users can obtain major inducements of sex reversal in this species in the SPECIES module. For any SRG, users can obtain its regulators, targets during sex determination in the SRG module and spatial distribution in different stages in the IMAGE module. For any annotated gene, users can obtain its homologous genes and conserved motifs in 18 species in the GENE module. Furthermore, users can also explore and visualize expression dynamics 
across different conditions in the GENE or BROWSER modules.

In the future, we will continuously select important and typical sex reversal species as their complete genome and omics data from both "female" and "male" samples become available. Hermaphroditic fish such as Synbranchus marmoratus and Amphiprion perideraion and invertebrates such as Macrobrachium rosenbergii and Venus mercenaria are candidates. In addition, we will add more omics data, such as sRNA-seq, BS-seq and ChIP-seq data. We expect that the resources in ASER will promote further studies to decode the molecular mechanisms of sex reversal.

\section{Data availability}

ASER is publicly available at http://aser.ihb.ac.cn/.

\section{CRediT author statement}

Yangyang Li: Software, Formal analysis, Data curation, Writing - original draft, Writing - review and editing. Zonggui Chen: Software, Formal analysis, Visualization, Writing - review and editing. Hairong Liu: Investigation, Data curation. Writing - review and editing. Qiming Li: Formal analysis. Xing Lin: Data curation. Shuhui Ji: Data curation. Rui Li: Data curation. Shaopeng Li: Data curation. Weiliang Fan: Visualization. Haiping Zhao: Data curation. Zuoyan Zhu: Writing review \& editing. Wei Hu: Conceptualization, Funding acquisition, Writing - review \& editing. Yu Zhou: Conceptualization, Project administration, Funding acquisition, Methodology, Writing - review and editing. Daji Luo: Conceptualization, Project administration, Funding acquisition, Methodology, Writing - review and editing. All authors read and approved the final manuscript.

\section{Competing interests}

The authors declare no conflict of interest. 


\section{Acknowledgements}

326 This work was supported by grants from the National Natural Science Foundation of 327 China (31922085, 31872191 to DL, 31922039 to YZ), the Strategic Priority Research 328 Program of CAS (XDA24010108) to WH and DL, and Natural Science Foundation of 329 of Hubei Province (2020CFA056 to DL and 2020CFA057 to YZ). Part of the 330 computation of this work was done in the Supercomputing Center of Wuhan 331 University and Hydrobiological Data Analysis Center.

\section{ORCID}

334 0000-0002-8441-8484 (Yangyang Li)

335 0000-0003-3346-2765 (Zonggui Chen)

336 0000-0002-6458-1786 (Hairong Liu)

337 0000-0003-4183-9913 (Qiming Li)

338 0000-0002-8471-6684 (Xing Lin)

339 0000-0002-4696-9330 (Shuhui Ji)

340 0000-0002-4434-2120 (Rui Li)

341 0000-0001-6415-2562 (Shaopeng Li)

342 0000-0003-4570-7686 (Weiliang Fan)

343 0000-0002-3982-3624 (Haiping Zhao)

344 0000-0002-6475-6427 (Wei Hu)

345 0000-0002-2102-9377 (Yu Zhou)

346 0000-0002-8051-9624 (Daji Luo)

\section{$348 \quad$ References}

349 [1] Shao C, Li Q, Chen S, Zhang P, Lian J, Hu Q, et al. Epigenetic modification and 350 inheritance in sexual reversal of fish. Genome Res 2014;24:604-15.

351 [2] Budd AM, Banh QQ, Domingos JA, Jerry DR. Sex Control in Fish: Approaches, 352 Challenges and Opportunities for Aquaculture. Journal of Marine Science and 353 Engineering 2015;3:329-55. 
354 [3] Mei J, Gui JF. Genetic basis and biotechnological manipulation of sexual

355 dimorphism and sex determination in fish. Sci China Life Sci 2015;58:124-36.

356 [4] Weber C, Capel B. Sex reversal. Curr Biol 2018;28:R1234-R6.

357 [5] Devlin RH, Nagahama Y. Sex determination and sex differentiation in fish: an 358 overview of genetic, physiological, and environmental influences. Aquaculture $3592002 ; 208: 191-364$.

360 [6] Holleley CE, O'Meally D, Sarre SD, Marshall Graves JA, Ezaz T, Matsubara K, et 361 al. Sex reversal triggers the rapid transition from genetic to temperature-dependent 362 sex. Nature 2015;523:79-82.

363 [7] Baroiller JF, D'Cotta H. Environment and sex determination in farmed fish. Comp 364 Biochem Physiol C Toxicol Pharmacol 2001;130:399-409.

365 [8] Todd EV, Liu H, Muncaster S, Gemmell NJ. Bending Genders: The Biology of 366 Natural Sex Change in Fish. Sex Dev 2016;10:223-41.

367 [9] Chen HJ, Liu HR, Li R, Lin X, Luo HR, Ji SH, et al. Blood cell identification and 368 hematological analysis during natural sex reversal in rice field eel (Monopterus albus). 369 Aquaculture 2021;538.

370 [10] Thomas JT, Todd EV, Muncaster S, Lokman PM, Damsteegt EL, Liu H, et al. 371 Conservation and diversity in expression of candidate genes regulating 372 socially-induced female-male sex change in wrasses. PeerJ 2019;7:e7032.

373 [11] Capel B. Vertebrate sex determination: evolutionary plasticity of a fundamental 374 switch. Nat Rev Genet 2017;18:675-89.

375 [12] Liu J, Liu X, Jin C, Du X, He Y, Zhang Q. Transcriptome Profiling Insights the 376 Feature of Sex Reversal Induced by High Temperature in Tongue Sole Cynoglossus 377 semilaevis. Front Genet 2019;10:522.

378 [13] Holleley CE, Sarre SD, O'Meally D, Georges A. Sex Reversal in Reptiles: 379 Reproductive Oddity or Powerful Driver of Evolutionary Change? Sex Dev $380 \quad 2016 ; 10: 279-87$.

381 [14] Crews D, Cantu AR, Rhen T, Vohra R. The relative effectiveness of estrone, 382 estradiol-17 beta, and estriol in sex reversal in the red-eared slider (Trachemys 383 scripta), a turtle with temperature-dependent sex determination. Gen Comp 
384

385

386

387

388

389

390

391

392

393

394

395

396

397

398

399

400

401

402

403

404

405

406

407

408

409

410

411

412

413

Endocrinol 1996;102:317-26.

[15] Major AT, Smith CA. Sex Reversal in Birds. Sex Dev 2016;10:288-300.

[16] Nakamura M. Is a sex-determining gene(s) necessary for sex-determination in amphibians? Steroid hormones may be the key factor. Sex Dev 2013;7:104-14.

[17] Wallace H, Badawy GM, Wallace BM. Amphibian sex determination and sex reversal. Cell Mol Life Sci 1999;55:901-9.

[18] Quinn A, Koopman P. The molecular genetics of sex determination and sex reversal in mammals. Semin Reprod Med 2012;30:351-63.

[19] Camerino G, Parma P, Radi O, Valentini S. Sex determination and sex reversal. Curr Opin Genet Dev 2006;16:289-92.

[20] Harding SD, Armit C, Armstrong J, Brennan J, Cheng Y, Haggarty B, et al. The GUDMAP database - an online resource for genitourinary research. Development 2011;138:2845-53.

[21] Lee TL, Li YM, Cheung HH, Claus J, Singh S, Sastry C, et al. GonadSAGE: a comprehensive SAGE database for transcript discovery on male embryonic gonad development. Bioinformatics 2010;26:585-6.

[22] Darde TA, Lecluze E, Lardenois A, Stevant I, Alary N, Tuttelmann F, et al. The ReproGenomics Viewer: a multi-omics and cross-species resource compatible with single-cell studies for the reproductive science community. Bioinformatics 2019;35:3133-9.

[23] Bradford Y, Conlin T, Dunn N, Fashena D, Frazer K, Howe DG, et al. ZFIN: enhancements and updates to the Zebrafish Model Organism Database. Nucleic Acids Res 2011;39:D822-9.

[24] Szklarczyk D, Morris JH, Cook H, Kuhn M, Wyder S, Simonovic M, et al. The STRING database in 2017: quality-controlled protein-protein association networks, made broadly accessible. Nucleic Acids Research 2017;45:D362-D8.

[25] Dobin A, Davis CA, Schlesinger F, Drenkow J, Zaleski C, Jha S, et al. STAR: ultrafast universal RNA-seq aligner. Bioinformatics 2013;29:15-21.

[26] Li H, Handsaker B, Wysoker A, Fennell T, Ruan J, Homer N, et al. The Sequence Alignment/Map format and SAMtools. Bioinformatics 2009;25:2078-9. 
414 [27] Pertea M, Kim D, Pertea GM, Leek JT, Salzberg SL. Transcript-level expression 415 analysis of RNA-seq experiments with HISAT, StringTie and Ballgown. Nat Protoc $416 \quad 2016 ; 11: 1650-67$.

417 [28] Love MI, Huber W, Anders S. Moderated estimation of fold change and 418 dispersion for RNA-seq data with DESeq2. Genome Biol 2014;15:550.

419 [29] Buchfink B, Xie C, Huson DH. Fast and sensitive protein alignment using 420 DIAMOND. Nat Methods 2015;12:59-60.

421 [30] Camacho C, Coulouris G, Avagyan V, Ma N, Papadopoulos J, Bealer K, et al. 422 BLAST+: architecture and applications. BMC Bioinformatics 2009;10:421.

423 [31] Emms DM, Kelly S. OrthoFinder: phylogenetic orthology inference for 424 comparative genomics. Genome Biol 2019;20:238.

425 [32] Bailey TL, Johnson J, Grant CE, Noble WS. The MEME Suite. Nucleic Acids 426 Res 2015;43:W39-49.

427 [33] Gupta S, Stamatoyannopoulos JA, Bailey TL, Noble WS. Quantifying similarity 428 between motifs. Genome Biol 2007;8:R24.

429 [34] Emms DM, Kelly S. STAG: Species Tree Inference from All Genes. bioRxiv 4302018.

431 [35] Emms DM, Kelly S. STRIDE: Species Tree Root Inference from Gene 432 Duplication Events. Mol Biol Evol 2017;34:3267-78.

433 [36] Kent WJ. BLAT--the BLAST-like alignment tool. Genome Res 2002;12:656-64.

434 [37] Harris R. Improved pairwise alignment of genomic dna. PhD thesis 2007.

435 [38] Blanchette M, Kent WJ, Riemer C, Elnitski L, Smit AF, Roskin KM, et al. 436 Aligning multiple genomic sequences with the threaded blockset aligner. Genome Res $437 \quad 2004 ; 14: 708-15$.

438 [39] Ramani R, Krumholz K, Huang YF, Siepel A. PhastWeb: a web interface for 439 evolutionary conservation scoring of multiple sequence alignments using phastCons 440 and phyloP. Bioinformatics 2019;35:2320-2.

441 [40] Ortega-Recalde O, Goikoetxea A, Hore TA, Todd EV, Gemmell NJ. The Genetics 442 and Epigenetics of Sex Change in Fish. Annu Rev Anim Biosci 2020;8:47-69. 
A

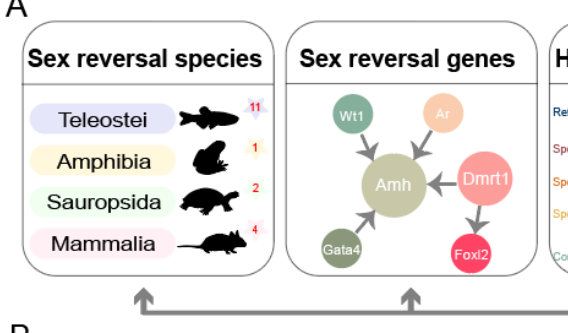

B

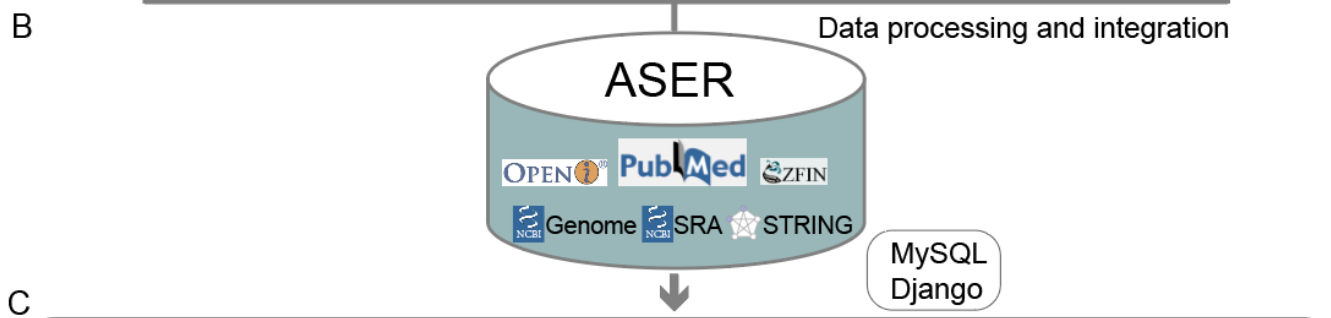

Figure 1
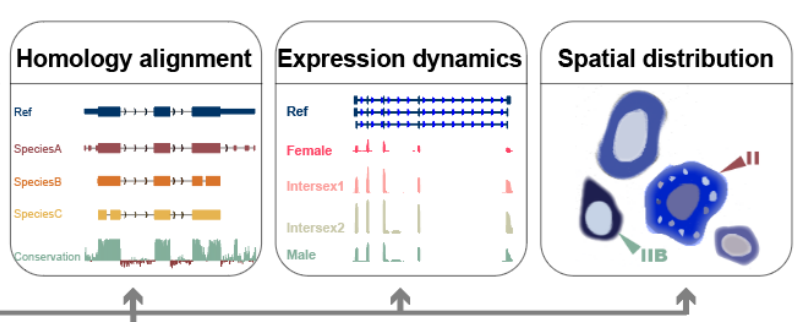

Data processing and integration

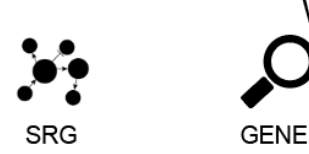

Web interface

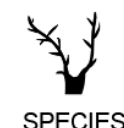

SPECIES

SRG
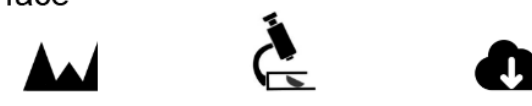

BROWSER

IMAGE

DOWNLOAD

449

Figure 1 Schematic diagram of ASER database

451 A. Five main functional modules in ASER, including the 18 sex reversal species, sex reversal genes (SRGs) and their regulatory networks, multiple sequence alignments and conservation scores, gene expression dynamics during sex reversal from RNA-seq data, spatial distribution of SRGs from ISH, FISH and ICH images. B. Data sources in ASER database. ASER stores all processed data in a MySQL database with additional indexes and uses the Django framework for interactive queries from the web interface to the backend database. C. Overview of the ASER web interface. The main functionalities are provided and organized into six modules. 
Figure 2

A

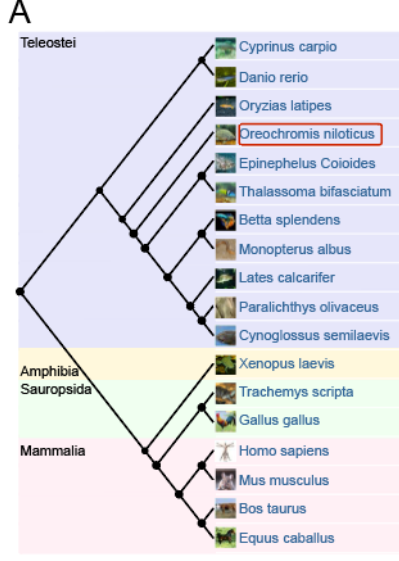

C

NAT GA HID TEM SF

\section{$B$}

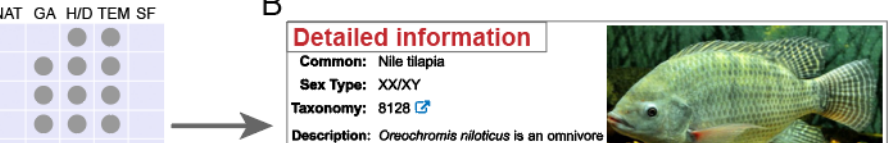

Genome information

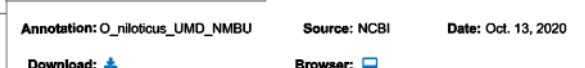

Download: $4 \quad$ Browser: $\square$

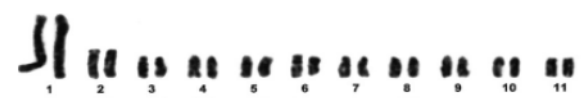

Sex reversal inducement Inducement

Temperature preference of Nile tilapia (Oreochromis niloticus) temperature

juveniles induces spontaneous sex reversal.

Brief exposure of embryos to steroids or aromatase inhibitor hormone, drug
induces sex reversal in Nile tilapia (Oreochromis nilloticus).

induces sex reversal in Nile tlapia (Oreochromis niloticus).

$\begin{array}{ll}\text { Mutation of foxl2 or cyp 19a1a Results in Female to Male Sex } & \text { genetic } \\ \text { Reversal in XX Nile Tilapia. } & \text { abnormality }\end{array}$

D

Danio rerio (Zebrafish)
Differentiative stage

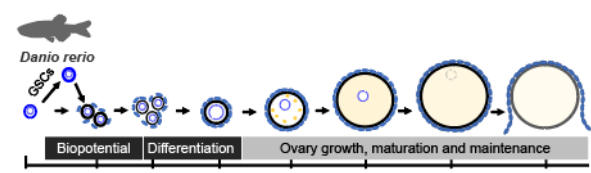

Differentiated

Species: Danio rerio

Developmental stage: adults

Differentiative stage: differentiated

Condition: natural

Gonad: ovary

Gene: cyp26a1

Description:

Expression of cyp26a1 was restricted to the ooplasm of oocytes and was not detected in somatic cells. In oocytes, cyp26a1 expression varied according to the stage of meiosis: it was barely detectable in early stage IB oocytes (elB, yellow arrowhead in F, prior to the diplotene stage of meiosis), was up-regulated in late stage IB oocytes that entered meiotic arrest at diplotene stage (IIB, green arrowhead in F), was maintained in stage II (red arrowhead in F) .....

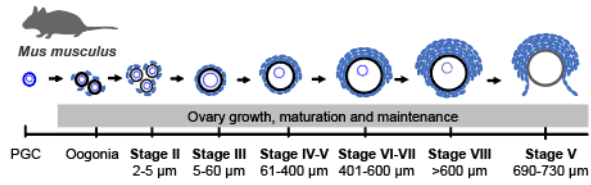

Reference:

Retinoic acid metabolic genes, meiosis, and gonadal sex differentiation in zebrafish

461 Figure 2 Species and image modules in ASER

462 A. Evolutionary relationship and sex reversal inducements of the 18 species belonging

463 to Teleostei, Mammalia, Sauropsida, and Amphibia. NAT: Natural; GA: Genetic 464 abnormality; H/D: Hormones or drugs; TEM: Temperature; SF: Social factors. B. 465 Detailed description, genome information, and references related to sex reversal in 466 each species. C. Examples of gonadal morphology at different developmental stages 467 in mouse and zebrafish. D. Image page describing the spatial distribution of SRGs in 468 gonads, with a representative example of cyp26a1 in ovaries. 
Figure 3

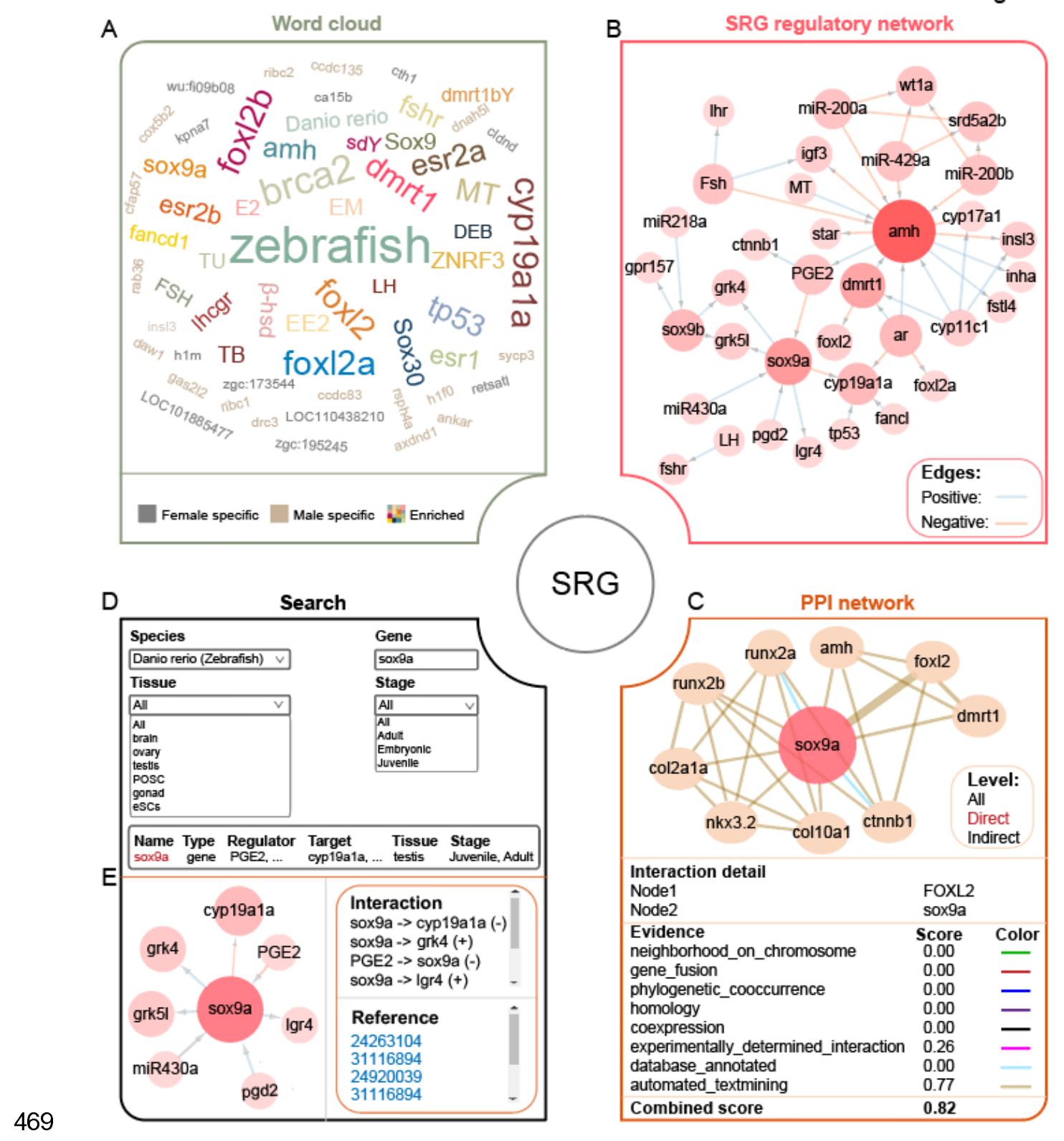

B SRG regulatory network

470 Figure 3 SRG module in ASER

471 A. Word cloud for genes or drugs mined from the literature as well as the top 50 genes

472 that are specifically expressed in females or males. The size represents word

473 frequency. B. Representative regulatory network of SRGs in zebrafish. The size

474 represents the node degree in the network. C. An interactive view of the

475 Protein-Protein Interaction (PPI) network for the SRG query. D. SRG regulatory

476 network search page by species, tissue, gene and stage. E. Detailed information,

477 regulatory relationships, and literature evidence for the SRG query. 
A

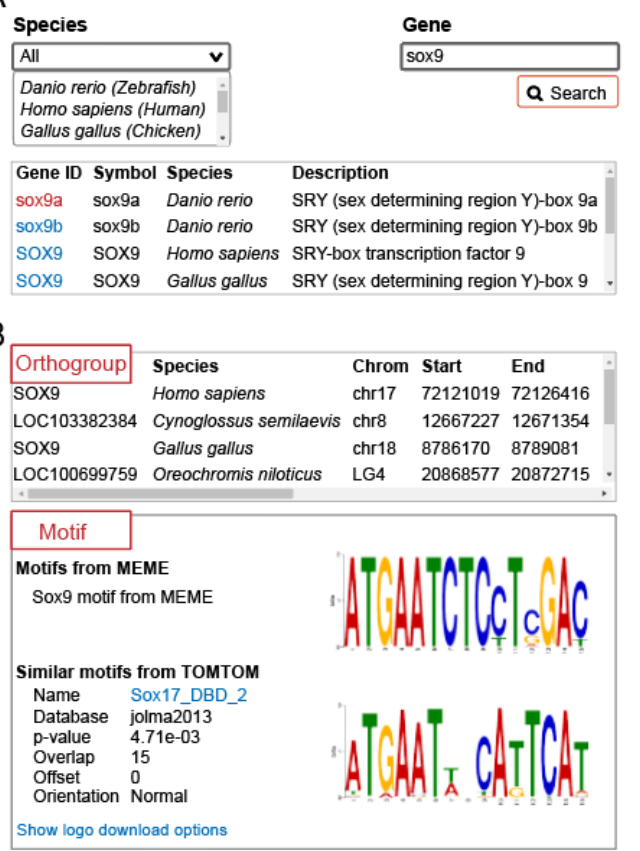

C

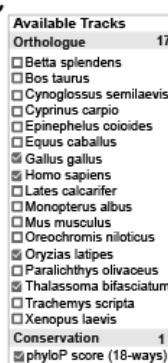

478
D

Figure 4

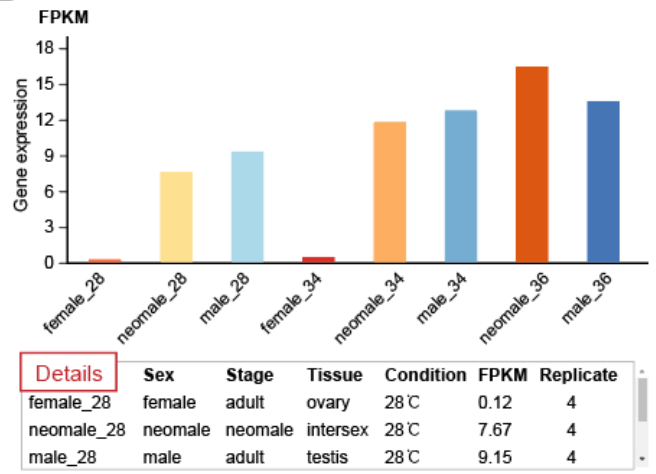

E

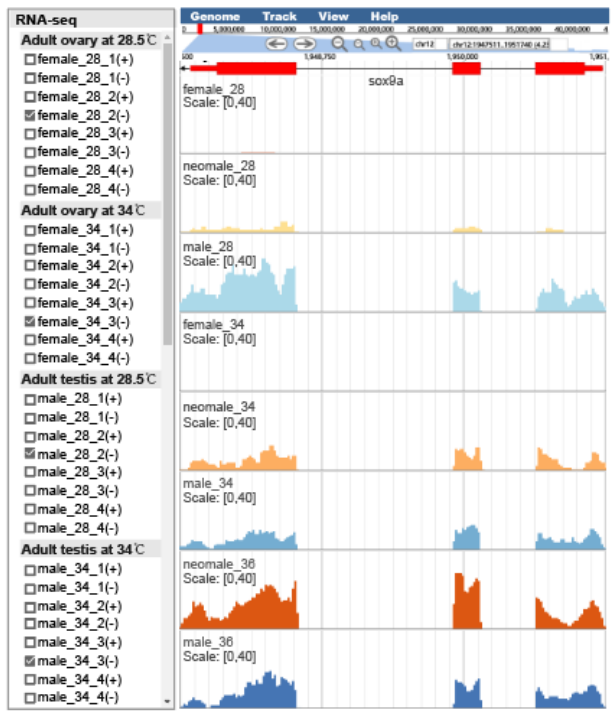

Figure 4 Gene conservation and expression modules in ASER

A. Search page for any gene in the 18 species. B. Homologous genes in the 18 species (top) and motifs corresponding to the query gene (bottom). The conserved motif for these homologous genes was computationally identified by MEME, and known motifs similar to this motif were also presented. C. Genome browser tracks of orthologue and conservation scores (18-ways). D. Expression dynamics of representative query gene. E. Genome browser view of processed RNA-seq signals for a representative query gene (sox9a in zebrafish). 
$491 \quad$ Tables

492 Table 1 Inducements of sex reversal or common approaches used to manipulate $493 \quad$ sex in 18 species

\begin{tabular}{|c|c|c|}
\hline Species & Gonadal differentiation & Inducements/Approaches \\
\hline Cyprinus carpio & gonochoristic & H/D; TEM \\
\hline Danio rerio & gonochoristic & GA; H/D; TEM \\
\hline Oryzias latipes & gonochoristic & GA; H/D; TEM \\
\hline Oreochromis niloticus & gonochoristic & GA; H/D; TEM \\
\hline Epinephelus coioides & hermaphroditic & NAT; GA; H/D; SF \\
\hline Thalassoma & hermaphroditic & NAT; SF \\
\hline \multicolumn{3}{|l|}{ bifasciatum } \\
\hline Betta splendens & gonochoristic & $\mathrm{H} / \mathrm{D}$ \\
\hline Monopterus albus & hermaphroditic & NAT \\
\hline Lates calcarifer & hermaphroditic & NAT \\
\hline Paralichthys olivaceus & gonochoristic & H/D; TEM \\
\hline Cynoglossus semilaevis & gonochoristic & NAT; TEM \\
\hline Xenopus laevis & gonochoristic & $\mathrm{H} / \mathrm{D}$ \\
\hline Trachemys scripta & gonochoristic & H/D; TEM; GA \\
\hline Gallus gallus & gonochoristic & $\mathrm{H} / \mathrm{D}$ \\
\hline Homo sapiens & gonochoristic & GA \\
\hline Mus musculus & gonochoristic & GA \\
\hline Bos taurus & gonochoristic & GA \\
\hline Equus caballus & gonochoristic & GA \\
\hline
\end{tabular}

494 


\section{Supplementary material}

A

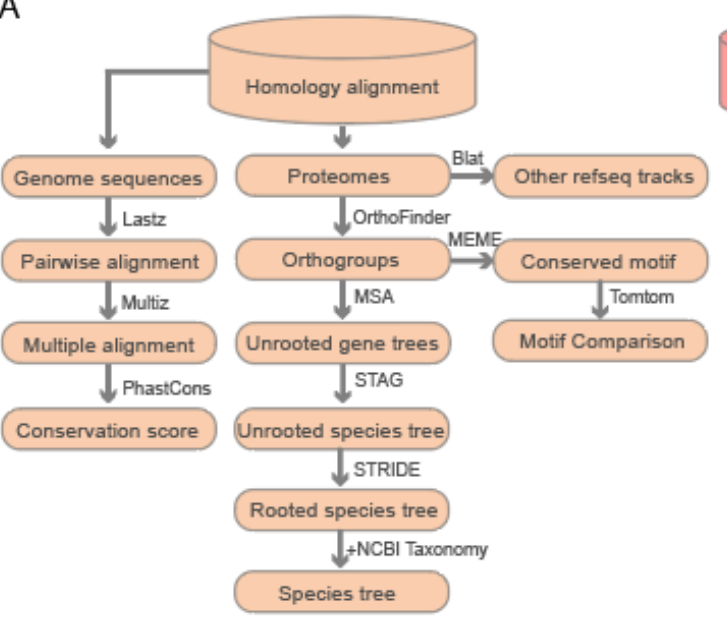

Supplementary Figure S1

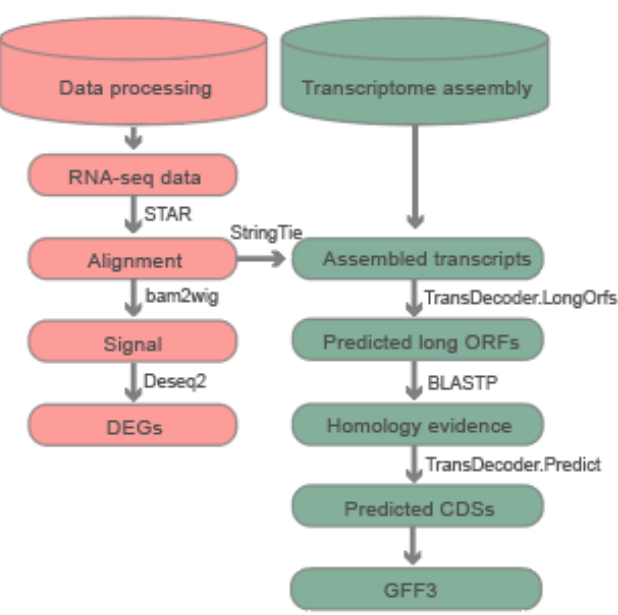

B

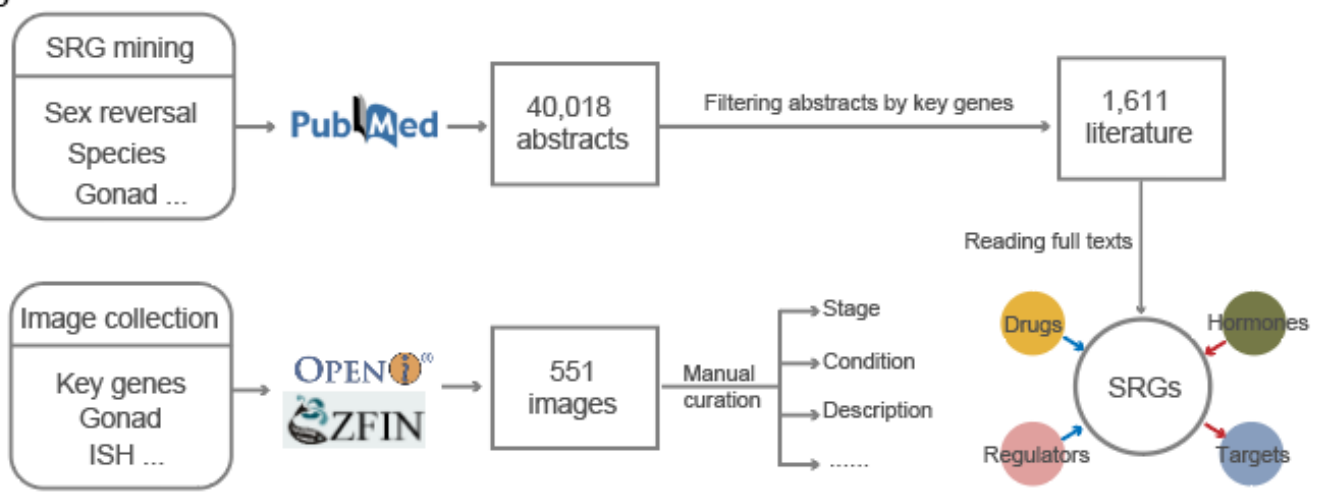

C Literature (258) Prediction (498)

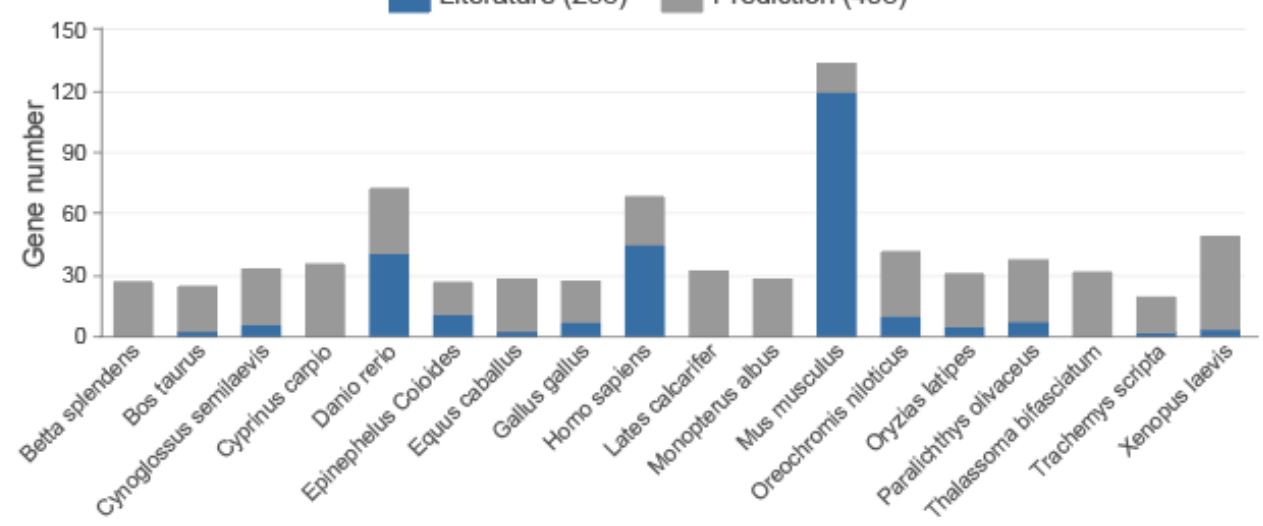

Figure S1 Data processing pipelines and statistics of SRGs

504 A. Workflows for building ASER, including homology alignment, RNA-seq data 505 processing, and transcriptome assembly. B. Workflows for SRG mining, and image 
506 collection and annotation. C. Statistics of validated SRGs and predicted genes

507 associated with sex reversal in the ASER database.

508

509 Tables

510 Table S1 RNA-seq data used in the ASER database

511 Table S2 Wordlist and blacklist in SRG mining

512 Table S3 Literature mining of SRGs for the ASER database

513 